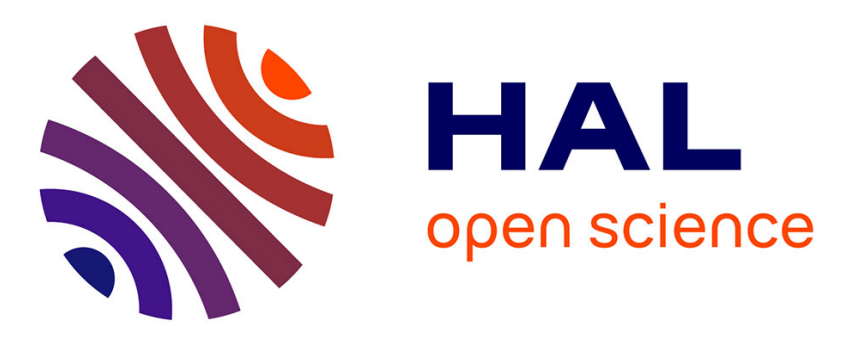

\title{
Deterministic Blind Separation of Sources having Different Symbol Rates using Tensor-Based Parallel Deflation
}

\author{
André L. F. de Almeida, Pierre Comon, Xavier Luciani
}

\section{To cite this version:}

André L. F. de Almeida, Pierre Comon, Xavier Luciani. Deterministic Blind Separation of Sources having Different Symbol Rates using Tensor-Based Parallel Deflation. Ninth International Conference on Latent Variable Analysis and Signal Separation, Sep 2010, Saint Malo, France. pp.362-369. hal00540504

\author{
HAL Id: hal-00540504 \\ https://hal.science/hal-00540504
}

Submitted on 26 Nov 2010

HAL is a multi-disciplinary open access archive for the deposit and dissemination of scientific research documents, whether they are published or not. The documents may come from teaching and research institutions in France or abroad, or from public or private research centers.
L'archive ouverte pluridisciplinaire HAL, est destinée au dépôt et à la diffusion de documents scientifiques de niveau recherche, publiés ou non, émanant des établissements d'enseignement et de recherche français ou étrangers, des laboratoires publics ou privés. 


\title{
Deterministic Blind Separation of Sources Having Different Symbol Rates Using Tensor-Based Parallel Deflation
}

\author{
André L. F. de Almeida ${ }^{(1)}$, Pierre Comon ${ }^{(2)}$, and Xavier Luciani ${ }^{(3)}$ \\ ${ }^{(1)}$ Wireless Telecom Research Group, Department of Teleinformatics Engineering, \\ Federal University of Ceará, CP 6005, Campus do Pici, 60455-760, Fortaleza, Brazil. \\ ${ }^{(2)}$ Laboratoire d'Informatique, Signaux et Systèmes de Sophia-Antipolis, UMR6070, \\ UNSA CNRS, 2000, route des Lucioles, Les Algorithmes, bât. Euclide B, BP 121, \\ 06903 Sophia Antipolis Cedex, France. \\ ${ }^{(3)}$ Laboratoire Traitement du Signal et de l'Images, Université de Rennes 1, U642 \\ INSERM, Campus de Beaulieu, bât. 22, 35042 Cedex, Rennes, France. \\ andre@gtel.ufc.br, , pcomon@i3s.unice.fr, , lucianix@gmail.com
}

\begin{abstract}
In this work, we address the problem of blind separation of non-synchronous statistically independent sources from underdetermined mixtures. A deterministic tensor-based receiver exploiting symbol rate diversity by means of parallel deflation is proposed. By resorting to bank of samplers at each sensor output, a set of third-order tensors is built, each one associated with a different source symbol period. By applying multiple Canonical Decompositions (CanD) on these tensors, we can obtain parallel estimates of the related sources along with an estimate of the mixture matrix. Numerical results illustrate the bit-error-rate performance of the proposed approach for some system configurations.
\end{abstract}

Key words: Blind source separation, underdetermined mixtures, nonsynchronous sources, tensor decomposition, parallel deflation.

\section{Introduction}

Blind Source Separation (BSS) and Blind Identification (BI) of underdetermined mixtures have now become classical problems in signal processing and telecommunications. A large part of the related algorithms resorts to Higher Order statistics, notably by exploiting the multilinear properties of cumulant tensors. For instance, FOOBI-1 and FOOBI-2 [1] algorithms are based on fourth order statistics whereas the 6-BIOME algorithm (also referred to as BIRTH) [2] relies on sixth order cumulants.

In many applications, the sources are known to be cyclostationnary. Indeed, this property appears as soon as digital communication signals are oversampled. In particular, the behavior of second- and fourth-order BSS algorithms in a cyclostationary context has been addressed in [3]. A partially unbiased estimator of the sixth-order cumulant tensor has been later proposed in [4] by taking 
into account the knowledge of the source cyclic frequencies. More generally, algorithms exploiting the cyclostationarity property resort to statistical tools.

In this study, we address the problem of underdetermined BSS and BI in the cyclostationary context by using a deterministic tensor-based receiver. Several methods have been proposed in the literature in order to solve BI and BSS problem using different multilinear models $[5,7,8]$. All of these works assume synchronous sources. This assumption is often unrealistic in non-cooperative systems (e.g. interception). The proposed receiver assumes non-synchronous sources. It consists of using a bank of sampling chains at each receive sensor, each one being adapted to the symbol rate of a given source, which are assumed to be known or estimated. At the output of the sampling stage, a set of third-order tensors is built, each one associated with a different sampling rate. As a consequence, we can obtain as many tensors as pre-detected sources. By applying successive canonical decompositions (CanD) [9] of these tensors, we obtain successive estimates of the related sources along with a mixture estimate. This approach can be connected to the parallel deflation method proposed in $[10,11]$.

This paper is organized as follows. In Section 2, we introduce the system model. In Section 3, a tensor-based formulation of the received data model is given and the proposed parallel deflation receiver is presented. Section 4 presents our simulation results and the paper is concluded in Section 5 .

\section{System Model}

Let us consider a uniform linear array of $I$ sensors receiving signals from $P$ sources. Let $\left\{s_{p}(t)\right\}$ be the information-bearing signal of the $p$-th source, $a_{i, p}$ be the sensor response of the $i$-th sensor to the $p$-th source, and $h_{p}(t)$ the timedomain signature of the $p$-th source, which comprises channel fading, transmit and receive filter responses. The baseband-equivalent signal received at the $i$-th sensor can be written, in absence of noise, as:

$$
y_{i}(t)=\sum_{p=1}^{P} a_{i, p} x_{p}(t)
$$

where $x_{p}(t)=\sum_{n=0}^{N-1} h_{p}\left(t-n T_{p}\right) s_{p}(n)$. Suppose that the $P$ sources have different symbol rates $1 / T_{p}$, where $T_{p}$ is the symbol period of the $p$-th source. By sampling the received signal $\left\{y_{p}(t)\right\}$ at a reference sampling rate $1 / T_{s}$, we obtain:

$$
y_{i}\left(m T_{s}\right)=\sum_{p=1}^{P} a_{i, p}\left(\sum_{n=0}^{N-1} h_{p}\left(m T_{s}-n T_{p}\right) s_{p}\left(n T_{s}\right)\right),
$$

where $m=0, \ldots, M-1$, and $M$ corresponds to the number of collected samples of the received signal. We assume that $h_{p}(t)$ is zero outside an interval $\left[0, K_{p}\right)$, $K_{p}<T_{p}, p=1, \ldots, P$, which means that the channel of each of the sources has no temporal dispersion, i.e. we have an instantaneous mixture of sources, 
or a flat-fading channel in communications terminology. Let us assume that the source symbol periods are organized in increasing order, i.e. $T_{s}=T_{1}<T_{2}<$ $\ldots<T_{P}$, where the symbol period of the first source is assumed to be equal to the reference sampling period. The received signal samples are collected during a fixed observation window of $M T_{s}$ seconds, regardless of the used sampling period. Therefore, when sampling at a rate $1 / T_{j}$ associated with the $j$-th source, the number $M_{j}$ of collected received signal samples is given by:

$$
M_{j}=\left\lceil M\left(\frac{T_{s}}{T_{j}}\right)\right\rceil=\left\lceil\frac{M}{\alpha_{j}}\right\rceil, \quad \alpha_{j} \in \mathbb{R}^{+} .
$$

Now, suppose that the sampling rate $1 / T_{s}$ at the receiver is $L$ times higher than the $p$-th source symbol rate, i.e. $T_{s}=T_{p} / L$. This means that the received data is oversampled by a factor of $L$ w.r.t. the $p$-th source. Then, $(2)$ is equivalent to the following model:

$$
y_{i}\left(\left(m_{j}+l / L\right) T_{j}\right)=\sum_{p=1}^{P} a_{i, p} h_{p}\left(l T_{j} / L\right) s_{p}\left(m_{j} T_{j}\right),
$$

$m_{j}=0, \ldots, M_{j}-1, l=0, \ldots, L-1, j=1, \ldots, P$. Suppressing the term $T_{j},(4)$ can be simplified to:

$$
y_{i}^{(j)}\left(m_{j}+l / L\right)=\sum_{p=1}^{P} a_{i, p} h_{p}^{(j)}(l / L) s_{p}^{(j)}\left(m_{j}\right),
$$

where $y_{i}^{(j)}\left(m_{j}+l / L\right) \doteq y_{i}\left(\left(m_{j}+l / L\right) T_{j}\right), h_{p}^{(j)}(l / L) \doteq h_{p}\left(l T_{j} / L\right)$, and $s_{p}^{(j)}\left(m_{j}\right) \doteq$ $s_{p}\left(m T_{j}\right)$. Note that $h_{p}^{(j)}(l / L)$ is the $l$-th polyphase component of the $p$-th source channel impulse response obtained by resampling the original channel impulse response at a factor of $L$ times the $j$-th source symbol rate. Likewise, $s_{p}^{(j)}\left(m_{j}\right)$ is obtained by resampling the $p$-th source symbol sequence at the $j$-th source symbol rate.

\section{Parallel Deflation Receiver}

In this section, we show that the received signal model given by (5) can be formulated as a third-order CanD model. Let $a_{i, p}, \bar{h}_{l, p}^{(j)} \doteq h_{p}^{(j)}((l-1) / L)$ and $\bar{s}_{m, p}^{(j)} \doteq s_{p}^{(j)}\left(m_{j}-1\right)$ be, respectively, the entries of the matrices $\mathbf{A} \in \mathbb{C}^{I \times P}$, $\overline{\mathbf{H}}^{(j)} \in \mathbb{C}^{L \times P}$, and $\overline{\mathbf{S}}^{(j)} \in \mathbb{C}^{M_{j} \times P}$ collecting sensor responses, channel coefficients and transmitted symbols. Define $\bar{y}_{i, m_{j}, l}^{(j)} \doteq y_{i}\left(\left(m_{j}+(l-1) / L\right) T_{j}\right)$ as the $\left(i, m_{j}, l\right)$ th entry of a third-order tensor $\overline{\mathcal{Y}}^{(j)} \in \mathbb{C}^{I \times M_{j} \times L}$ collecting the overall received signal sampled at a rate of $T_{j} / L, i \in[1, I], m_{j} \in\left[1, M_{j}\right], l \in[1, L]$. With these definitions, we can rewrite (4) as a CanD model:

$$
\bar{y}_{i, m_{j}, l}^{(j)}=\sum_{p=1}^{P} a_{i, p} \bar{s}_{m_{j}, p}^{(j)} \bar{h}_{l, p}^{(j)}, \quad j=1, \ldots, P,
$$


or, alternatively, in unfolded matrix forms:

$$
\begin{aligned}
& \overline{\mathbf{Y}}_{1}^{(j)}=\left(\overline{\mathbf{H}}^{(j)} \odot \overline{\mathbf{S}}^{(j)}\right) \mathbf{A}^{T} \in \mathbb{C}^{L M_{j} \times I} \\
& \overline{\mathbf{Y}}_{2}^{(j)}=\left(\mathbf{A} \odot \overline{\mathbf{H}}^{(j)}\right) \overline{\mathbf{S}}^{(j) T} \in \mathbb{C}^{I L \times M_{j}} \\
& \overline{\mathbf{Y}}_{3}^{(j)}=\left(\overline{\mathbf{S}}^{(j)} \odot \mathbf{A}\right) \overline{\mathbf{H}}^{(j) T} \in \mathbb{C}^{M_{j} I \times L},
\end{aligned}
$$

where $\odot$ denotes the Khatri-Rao (columnwise Kronecker) product.

Therefore, by performing $P$ sampling operations over the received signal, each one w.r.t. a different source symbol rate, we can construct $P$ different CanD tensors $\overline{\mathcal{Y}}^{(1)}, \ldots, \overline{\mathcal{Y}}^{(P)}$, each one of which will be associated with the detection of a given source. Note that only the $j$-th column of $\overline{\mathbf{S}}^{(j)}$ contains useful information (in this case it contains the $j$-th source symbol sequence). Due to the symbol rate mismatch between sources, the remaining $P-1$ columns of $\overline{\mathbf{S}}^{(j)}$ only correspond to structured noise generated by the corresponding sources.

The proposed method to blindly extract the source signals (as well as to estimate the sensor and channel responses), is to fit each of the received signal tensors $\overline{\mathcal{Y}}^{(1)}, \ldots, \overline{\mathcal{Y}}^{(P)}$ to its associated third-order CanD tensor model in the least squares (LS) sense using the unfolded factorizations (7)-(9). In this case, the tensor-based detection has $P$ processing stages. At each stage, we can use, for instance, the alternating least squares (ALS) algorithm, which consists of alternated estimations of the sensor, symbol and channel matrices according to the following criterion [5]:

$$
\min _{\left\{\widehat{\mathbf{A}}, \widehat{\overline{\mathbf{S}}}^{(j)}, \widehat{\mathbf{H}}^{(j)}\right\}}\left\|\overline{\mathcal{Y}}^{(j)}-\sum_{p=1}^{P} \mathbf{a}_{p} \circ \overline{\mathbf{s}}_{p}^{(j)} \circ \overline{\mathbf{h}}_{p}^{(j)}\right\|^{2}, \quad j=1, \ldots, P .
$$

Note that we need to process all the $P$ tensors $\overline{\mathcal{Y}}^{(1)}, \ldots, \overline{\mathcal{Y}}^{(P)}$ in order to extract the symbol sequence of all the sources. Of course, this is due to the fact that only the $j$-th source symbol sequence can be extracted from $\overline{\mathcal{Y}}^{(j)}$, the remaining symbol sequences appear only as structure interference due to source symbol rate mismatch.

\section{Uniqueness issue}

The CanD model (6) representing the received signal tensor $\overline{\mathcal{Y}}^{(j)} \in \mathbb{C}^{I \times M_{j} \times L}$ enjoys the essential uniqueness property, which means that $\left\{\mathbf{A}, \overline{\mathbf{S}}^{(j)}, \overline{\mathbf{H}}^{(j)}\right\}$ and $\left\{\widehat{\mathbf{A}}, \widehat{\mathbf{S}}^{(j)}, \widehat{\mathbf{H}}^{(j)}\right\}$ giving rise to the same tensor $\overline{\mathcal{Y}}^{(j)}$ are linked by the following relations $\widehat{\mathbf{A}}=\mathbf{A} \boldsymbol{\Pi} \boldsymbol{\Delta}_{A}, \widehat{\overline{\mathbf{S}}}^{(j)}=\mathbf{C} \boldsymbol{\Pi} \boldsymbol{\Delta}_{S}, \widehat{\hat{\mathbf{H}}}^{(j)}=\overline{\mathbf{H}}^{(j)} \boldsymbol{\Pi} \boldsymbol{\Delta}_{H}$, with $\boldsymbol{\Delta}_{A} \boldsymbol{\Delta}_{S} \boldsymbol{\Delta}_{H}=\mathbf{I}_{P}$, where $\boldsymbol{\Pi}$ is a permutation matrix whereas $\Delta_{A}, \Delta_{S}$, and $\boldsymbol{\Delta}_{H}$ are diagonal matrices. A sufficient condition for such an uniqueness was first established in [6] and generalized in [5] to the complex case. This condition states that the CanD model (6) is essentially unique if $k_{\mathbf{A}}+k_{\overline{\mathbf{S}}^{(j)}}+k_{\overline{\mathbf{H}}^{(j)}} \geq 2(P+1)$, where $k_{\mathbf{A}}$ denotes the Kruskal-rank, also called k-rank, of $\mathbf{A}$, i.e. the greatest integer $k_{\mathbf{A}}$ such that any set of $k_{\mathbf{A}}$ columns of $\mathbf{A}$ is linearly independent. The rank and the 


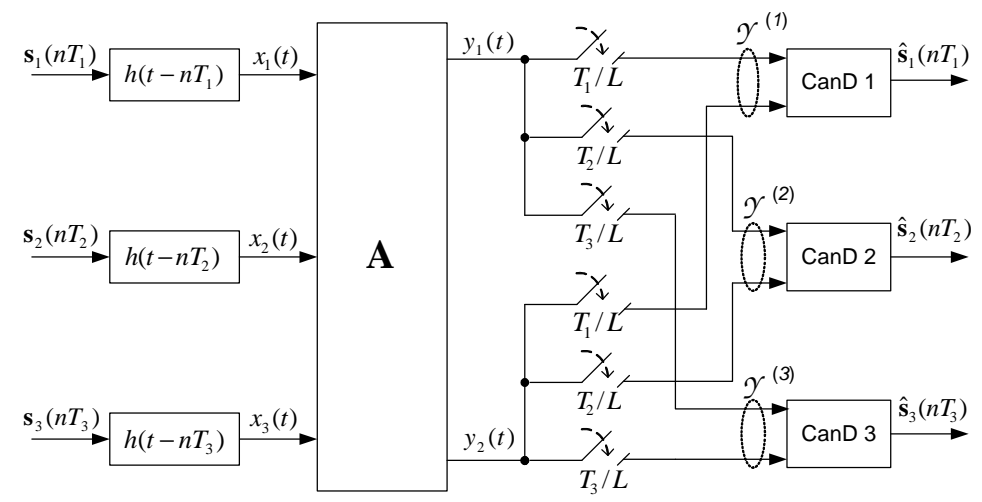

Fig. 1. Block-diagram of the proposed tensor-based parallel deflation receiver.

Kruskal-rank of $\mathbf{A}$ are related by the inequality $k_{\mathbf{A}} \leq \operatorname{rank}(\mathbf{A})$. Assuming that $\mathbf{A}, \overline{\mathbf{S}}^{(j)}$ and $\overline{\mathbf{H}}^{(j)}$ are full rank matrices, the Kruskal-rank and the rank coincide, so that

$$
\min (I, P)+\min \left(M_{j}, P\right)+\min (L, P) \geq 2(P+1)
$$

ensures the essential uniqueness of the CanD model (6) for the received signal tensor sampled at a rate of $T_{j} / L$.

In this work, we are interested in the case of underdetermined mixtures (i.e. more sources than sensors). According to condition (11), if $M_{j} \geq P$ and $L \geq P$, then $I=2$ sensors are enough to blindly separate $P$ sources.

\section{Tensor-based algorithm for parallel deflation}

* Here, we describe the $j$-th step of the algorithm which corresponds to the extraction of the $j$-th source. This process has to be repeated for $j=1, \ldots, P$ :

1. Resample the output signal of each sensor at the frequency $L / T_{j}$;

2. Build the three-way tensor $\overline{\mathcal{Y}}^{(j)} \in \mathbb{C}^{L \times M_{j} \times I}$ suitable for the $j$-th source;

3. Estimate loadings matrices $\widehat{\mathbf{A}}, \widehat{\widehat{\mathbf{S}}}^{(j)}, \widehat{\overline{\mathbf{H}}}^{(j)}$ describing the CanD of tensor $\overline{\mathcal{Y}}^{(j)}$ by minimizing criterion (10) in the LS sense (e.g. using the ALS algorithm [5]);

4. Evaluate and compare the "discrete" structure of each column of $\widehat{\widehat{\mathbf{S}}}^{(j)}=$ $\left[\widehat{\mathbf{s}}_{1}^{(j)}, \ldots, \widehat{\mathbf{s}}_{P}^{(j)}\right]$ in order to choose the suitable vector corresponding to the $j$-th source;

Remark 1: In addition to blind source separation, this algorithm also provides an estimation of the mixing matrix. A natural approach consists of estimating one column of matrix $\mathbf{A}$ at each deflation layer. Since only a single source is extracted at each detection layer, we eliminate the inherent column permutation ambiguity that would exist in a joint CanD estimation of equal symbol rate sources. The mixture identification problem will be addressed in a future work. 


\section{Performance Evaluation}

In this section, computer simulation results are provided for the performance evaluation of the proposed tensor-based deflation receiver in a wireless communication system. The receiver is equipped with a uniform linear array of halfwavelength spaced sensors. The propagation channel associated with each source is characterized by a complex envelope, an angle of arrival and a delay of arrival (assumed to be negligible with respect to the symbol source period). All these parameters are assumed to be constant during a data block of duration $M T_{s}$ seconds. At each sensor, the received signal is sampled at the Nyquist rate at the input of each deflation layer, i.e. $T_{e}=T_{j} / 2, j=1, \ldots, P$. The transmit/receive filters are raised cosines with roll-off factor 0.3 . We assume binary phase shift keying (BPSK) modulation for all the sources. The sources carrier residues are assumed to be negligible. Additive noise samples are modeled as complex Gaussian random variables with equal variance for all the sensors.

We compute the bit error rate (BER) for several signal-to-noise ratios (SNR) and for different system parameters (number of sources, number of sensors, sampling factor and data block size). The results are validated from 1000 MonteCarlo runs. Each run is associated with a different realization of the source symbol vectors, mixing matrix, noise tensor, angles of arrival, complex envelopes and the ratio of source symbol periods. The angles of arrival of the sources are randomly drawn between 0 and $80^{\circ}$ according to a uniform distribution. The symbol period $T_{1}$ of source 1 is taken as the reference sampling period, while those of the remaining sources $\left(T_{2}, \ldots, T_{P}\right)$ are randomly varied at each run are given by $T_{p}=T_{p}^{0} \pm \Delta_{p}, p=2, \ldots, P$, where $T_{p}^{0}$ is the median value of the $p$-th source symbol period, and $\Delta_{p}$ is a random variable uniformly distributed between $[0,1 / 2]$, which is assumed to be the same for all the sources. Our simulations suppose that $T_{1}, \ldots, T_{P}$ are known or have been estimated in a previous processing stage. The estimation of the symbol period is outside the scope of this paper. However, the methods proposed in $[12,13]$ can be applied in this context. We consider two underdetermined mixture setups: i) 3 sources and 2 sensors and ii) 4 sources et 3 sensors. We define $\overline{\mathcal{Y}}^{(j)} \in \mathbb{C}^{L \times M_{j} \times I}$ as the noise free data tensor built from the $j$-th sampler output $L / T_{j}$. The noise samples are stored in a tensor $\mathcal{N}^{(j)} \in \mathbb{C}^{L \times M_{j} \times I}$, so that the received data tensor $\tilde{\overline{\mathcal{Y}}}^{(j)}$ is given by $\tilde{\overline{\mathcal{Y}}}^{(j)}=\overline{\mathcal{Y}}^{(j)}+\mathcal{N}^{(j)}$. The SNR is defined as SNR $=10 \log _{10} \frac{\left\|\overline{\mathcal{Y}}^{(j)}\right\|_{F}^{2}}{\left\|\mathcal{N}^{(j)}\right\|_{F}^{2}} \quad(\mathrm{~dB})$, where the variance of $\mathcal{N}^{(j)}$ is computed in order to obtained the desired SNR level.

We first show the BER performance of the proposed receiver in the underdetermined case of 3 sources and 2 sensors $(P=3, I=2)$. The data block size is equal to 100 samples for source 1 (deflation layer 1 ). Since the length of the considered data block is fixed to $M=M_{1}=100$ for source 1 , the number of samples $M_{2}, \ldots, M_{P}$ processed by layers 2 to $P$ is lower than 100 and depends on the source symbol period ratios each run. The average BER values are computed over the 1000 runs and the $P$ sources. These values are reported in Table 1 for different SNR's and sampling factors. Herein, the source symbol periods are given by $T_{1}=T_{e}, T_{2}=\left(1.5 \pm \Delta_{2}\right) T_{e}, T_{3}=\left(2.5 \pm \Delta_{3}\right) T_{e}$, where $\Delta_{2}$ et $\Delta_{3}$ follow 


\begin{tabular}{|c|c|c|c|c|}
\hline SNR (dB) & \multicolumn{3}{|c|}{ Oversampling factor } \\
\hline & $L=3$ & $L=4$ & $L=6$ & $L=8$ \\
\hline \hline 0 & 0.2501 & 0.1868 & 0.1491 & 0.0688 \\
\hline 5 & 0.1633 & 0.0852 & 0.0855 & 0.0398 \\
\hline 10 & 0.1125 & 0.0553 & 0.0541 & 0.0334 \\
\hline 15 & 0.0957 & 0.0418 & 0.0409 & 0.0322 \\
\hline 20 & 0.0809 & 0.0390 & 0.0410 & 0.0294 \\
\hline
\end{tabular}

Table 1. Effect of sampling factor and SNR on the average BER $(P=3, I=2)$.

an uniform distribution in the range $[0,0.5]$. One can first note a significant degradation of the BER when the sampling factor is equal to 3. Better results can be obtained by increasing the sample factor. Only a slight improvement is observed for $L=4$ and $L=6$, although satisfying results are obtained for $L=8$. Table 2 compares the average BER values of the best estimated source with the worst estimated one. The data block size and the sampling factor are fixed to $N=100$ and $L=4$, respectively. We now consider two cases: $(P=3, I=2)$ and $(P=4, I=3)$. In the first case, the source symbol periods are $T_{1}=T_{e}$, $T_{2}=\left(1.5 \pm \Delta_{2}\right) T_{e}, T_{3}=\left(2.5 \pm \Delta_{3}\right) T_{e}$. In the second case, we have $T_{1}=T_{e}$, $T_{2}=\left(1.4 \pm \Delta_{2}\right) T_{e}, T_{3}=\left(1.8 \pm \Delta_{3}\right) T_{e}, T_{4}=\left(2.2 \pm \Delta_{4}\right) T_{e}$. We can note significant performance deviations between different deflation layers. One should note that the symbol period ratios between different sources are changed at each Monte Carlo run. Thus, when any two sources have very close symbol periods, their parallel extraction becomes more difficult and performance will be affected. With an eye to a reference comparison, we have also simulated the maximum-aposteriori (MAP) estimator based on the estimated channel, for $L=3, I=2$ and $\mathrm{SNR}=20 \mathrm{~dB}$. According to Table 3 the performance gap between both receivers is not significant for $(P=6, N=50)$ and $(P=6, N=100)$.

\begin{tabular}{|c|c|c|c|c|}
\hline SNR (dB) & \multicolumn{3}{|c|}{ System configuration } \\
\hline & \multicolumn{2}{|c|}{$P=3, I=2$} & \multicolumn{2}{c|}{$P=4, I=3$} \\
\hline & BER $_{\max }$ & BER $_{\min }$ & $\mathrm{BER}_{\max }$ & $\mathrm{BER}_{\text {min }}$ \\
\hline \hline 0 & 0.2222 & 0.1253 & 0.2637 & 0.1495 \\
\hline 5 & 0.1179 & 0.0427 & 0.1670 & 0.0620 \\
\hline 10 & 0.0837 & 0.0161 & 0.1383 & 0.0345 \\
\hline 15 & 0.0621 & 0.0121 & 0.1328 & 0.0332 \\
\hline 20 & 0.0587 & 0.0092 & 0.1421 & 0.0384 \\
\hline
\end{tabular}

Table 2. Average BER or the best estimated source $\left(\mathrm{BER}_{\max }\right)$ and for the worst estimated sources $\left(\mathrm{BER}_{\min }\right)$ computed over 1000 Monte-Carlo runs $(N=50, L=4)$.

\section{Conclusions and Perspectives}

We have proposed a new deterministic tensor-based receiver for blind source separation and channel identification in the case of underdetermined mixtures. The proposed receiver efficiently exploits symbol rate diversity and uses a CanDbased parallel deflation approach to individually extract each source. According 


\begin{tabular}{|l|c|c|c|}
\hline & \multicolumn{3}{|c|}{ Configuration } \\
\hline & $P=6, N=50$ & $P=6, N=100$ & $P=8, N=100$ \\
\hline \hline CanD deflation & 0.023 & 0.025 & 0.013 \\
\hline MAP estimator & 0.014 & 0.020 & 0.005 \\
\hline
\end{tabular}

Table 3. Comparison with the MAP estimator $(P=3, I=2, \mathrm{SNR}=25 \mathrm{~dB})$.

to our results, satisfactory performances can be obtained, especially when the source symbol rates are not too close. In addition to source separation, our approach allows to blindly estimate the full mixture matrix either jointly or separately for each source. Perspectives include comparisons with existing statistical BSS/BI approaches and a generalization to convolutive mixtures.

\section{References}

1. L. De Lathauwer, J. Castaing, and J-. F. Cardoso: Fourth-order cumulant-based blind identification of underdetermined mixtures. IEEE Trans. Signal Processing, 55(2):2965-2973, Feb. 2007.

2. L. Albera, A. Ferreol, P. Comon, and P. Chevalier: Blind identification of Overcomplete MixturEs of sources (BIOME). Linear Algebra Applications, Special Issue on Linear Algebra in Signal and Image Processing, 391C:3-30, Nov. 2004.

3. A. Ferreol and P. Chevalier, "On the behavior of current second and higher order blind source separation methods for cyclostationary sources," IEEE Trans. Sig. Proc., vol. 48, pp. 1712-1725, June 2000, erratum in vol.50, pp.990, Apr. 2002.

4. A. L. F. De Almeida, X. Luciani, P. Comon, "Blind identification of underdetermined mixtures based on the hexacovariance and higher-order cyclostationarity," In SSP'09, Cardiff, Wales, UK, Aug 31 - Sep. 3, 2009.

5. N. D. Sidiropoulos, G. B. Giannakis, and R. Bro: Blind PARAFAC receivers for DS-CDMA systems. IEEE Trans. Signal Process., 48(3):810-822, Mar. 2000.

6. J.B. Kruskal: Three-way arrays: rank and uniqueness of trilinear decompositions. with application to arithmetic complexity and statistics," Lin. Algebra Appl., 18:95$138,1977$.

7. A. L. F. De Almeida, G. Favier, and J. C. M. Mota: PARAFAC-based unified tensor modeling for wireless communication systems with application to blind multiuser equalization. Signal Processing, 87(2):337-351, Feb. 2007.

8. D. Nion and L. De Lathauwer: Block component model-based blind DS-CDMA receiver IEEE Trans. Signal Process., 56(11):5567-5579, November 2008.

9. P. Comon: Tensor Decompositions, State of the Art and Applications. In J. G. McWhirter and I. K. Proudler, Eds., Mathematics in Signal Processing V, pp. 1-24. Clarendon Press, Oxford, UK, 2002.

10. V. Zarzoso, L. Rota, and P. Comon: Deflation parallele avec des contrastes APF pour l'extraction aveugle de sources. In Gretsi'05, Louvain La Neuve, Belgique, 5-10, Sep. 2005.

11. L. Rota, V. Zarzoso, and P. Comon: Parallel deflation with alphabet-based criteria for blind source extraction. In IEEE SSP'05, Bordeaux, France, July, 17-20 2005.

12. S. Houcke, A. Chevreuil, and P. Loubaton: Blind equalization: case of an unknown symbol period. IEEE Trans. on Signal Processing, 51(3):781-793, Mar. 2003.

13. P. Jallon, A. Chevreuil, P. Loubaton, and P. Chevalier: Separation of convolutive mixtures of cyclostationary sources: a contrast function based approach. In Proc. ICA'04, Grenade, Spain, Sep. 2004. 